\title{
Low-Dose-Rate, Low-Dose Irradiation Delays Neurodegeneration in a Model of Retinitis
} Pigmentosa

\author{
Atsushi Otani, Hiroshi Kojima, Congrong Guo, \\ Akio Oishi, and Nagahisa Yoshimura \\ From the Department of Ophthalmology, Kyoto University \\ Graduate School of Medicine, Kyoto, Japan
}

The existence of radiation hormesis is controversial. Several stimulatory effects of low-dose (LD) radiation have been reported to date; however, the effects on neural tissue or neurodegeneration remain unknown. Here, we show that $L D$ radiation has a neuroprotective effect in mouse models of retinitis pigmentosa, a hereditary, progressive neurodegenerative disease that leads to blindness. Various LD radiation doses were administered to the eyes in a retinal degeneration mouse model, and their pathological and physiological effects were analyzed. LD gamma radiation in a low-dose-rate (LDR) condition rescues photoreceptor cell apoptosis both morphologically and functionally. The greatest effect was observed in a condition using $650 \mathrm{mGy}$ irradiation and a $26 \mathrm{mGy} /$ minute dose rate. Multiple rounds of irradiation strengthened this neuroprotective effect. A characteristic up-regulation (563\%) of antioxidative gene peroxiredoxin-2 (Prdx2) in the LDR-LD-irradiated retina was observed compared to the sham-treated control retina. Silencing the Prdx2 using small-interfering RNA administration reduced the LDR-LD rescue effect on the photoreceptors. Our results demonstrate for the first time that LDR-LD irradiation has a biological effect in neural cells of living animals. The results support that radiation exhibits hormesis, and this effect may be applied as novel therapeutic concept for retinitis pigmentosa and for other progressive neurodegenerative diseases regardless of the mechanism of degeneration involved. (Am J Pathol 2012, 180:328-336; DOI: 10.1016/j.ajpath.2011.09.025)

Hormesis refers to a process in which exposure to a low dose (LD) of an agent that is toxic at higher doses induces a beneficial effect on a cell or organism. This term has been widely used in the field of toxicology, where it is defined as "an adaptive response characterized by biphasic dose responses of generally similar quantitative features with respect to amplitude and range of the stimulatory response that are either directly induced or the result of compensatory biological processes following an initial disruption in homeostasis." ${ }^{1}$ Some researchers have raised the concept of "neurohormesis," which they define as the adaptive process of neurons and the nervous system responding to a moderate level of stress by enhancing their ability to resist a more severe stress that might otherwise be a lethal cause of dysfunction or disease. ${ }^{2}$ Examples of neurohormesis include ischemic preconditioning $^{3}$ and adaptive responses of neurons to moderate-intensity excitatory neurotransmission, ${ }^{4}$ exercise, ${ }^{5}$ and dietary restriction. ${ }^{6}$ Several endogenous neurotoxic molecules can induce neurohormesis, including nitric oxide, ${ }^{7}$ carbon monoxide, ${ }^{8}$ glutamate, ${ }^{9}$ and $\mathrm{Ca}^{2+} .{ }^{10}$

Biological responses to LD radiation are very different from those to high-dose (HD) radiation. HD ionizing radiation is generally known to be harmful to living organisms. However, LD radiation is not considered as harmful as once thought. The beneficial biological effects of LD radiation have been reported in vitro and in vivo. These effects include growth rate stimulation, ${ }^{11}$ survival enhancement after lethal HD irradiation, ${ }^{12}$ life span prolongation, ${ }^{13-15}$ immune function activation, ${ }^{16-18}$ improved behavior as demonstrated in mice, ${ }^{19}$ and disease prevention and cure. ${ }^{20-24}$ These advantageous effects of LD radiation are called radiation hormesis. ${ }^{25}$ However, radiation hormesis has been reported in only a few research fields, and the effects of LD radiation on neural tissue and neurodegeneration have not yet been elucidated.

Supported by the Ministry of Education, Science, Sports and Culture, Japan (grant-in-aid no. 17689045) and the Takeda Science Foundation. Accepted for publication September 14, 2011.

Supplemental material for this article can be found at http://ajp. amjpathol.org or at doi: 10.1016/j.ajpath.2011.09.025.

Address reprint requests to Atsushi Otani, M.D., Ph.D., Department of Ophthalmology, Kyoto University Graduate School of Medicine, 54-Kawaharacho, Shogoin, Sakyo-ku, Kyoto 606-8507, Japan. E-mail: otan@kuhp. kyoto-u.ac.jp. 
In the current study, we show that low-dose-rate (LDR)-LD radiation has a protective effect in both $r d 1$ and rd10 mouse models of retinitis pigmentosa (RP), a hereditary neurodegenerative disease of the retina that leads to blindness. We found that 650 mGy gamma LD radiation administered as LDR rescues rod photoreceptor cells from apoptosis and prevents the associated cone photoreceptor cell degeneration both morphologically and functionally. We also discovered that multiple rounds of irradiation dramatically increase photoreceptor survival compared with a single round of irradiation and also provides a long-term rescue effect. The polymerase chain reaction (PCR) array for the apoptosis pathway revealed that peroxiredoxin-2 ( $\operatorname{Prd} \times 2$ ) was highly up-regulated in the LDR-LD-irradiated retina. Prdx2 is an antioxidative gene ${ }^{26}$ that might play a neuroprotective role in neurodegenerative diseases. ${ }^{27,28}$ We found that LDR-LD irradiation induces Prdx2 expression in the photoreceptor cells of $r d$ mice and wild-type mice. Silencing the Prdx2 gene by small-interfering RNA (siRNA) blocked the rescue effect of photoreceptors by LDR-LD. Those studies strongly indicate that up-regulation of the antioxidative gene Prdx2 is one of the key molecularly based mechanisms for radiation-induced antiapoptotic action in retinal photoreceptor cells.

\section{Materials and Methods}

\section{Mouse Strains}

All animals were handled in accordance with the Association for Research of Vision and Ophthalmology statement for the use of animals in ophthalmic and vision research. The institutional review board of Kyoto University Graduate School of Medicine approved all experimental procedures. $\mathrm{C} 57 \mathrm{BL} / 6$ and $\mathrm{C} 3 \mathrm{H} / \mathrm{HeJ}(\mathrm{rd} 1 / \mathrm{rd} 1)$ mice were purchased from Japan SLC Inc. (Shizuoka, Japan). B6.CXB1-Pde6b $b^{r d 1} / J$ (rd10) mice were purchased from the Jackson Laboratory (Bar Harbor, ME). $\mathrm{C} 3 \mathrm{H} / \mathrm{HeJ}$ mice are homozygous for the rd1 mutation, which causes early onset and very rapid retinal degeneration. The mutation is in exon 7 of the Pde6b gene that encodes the rod photoreceptor cyclic guanosine monophosphate (cGMP) phosphodiesterase $\beta$-subunit. Mutations in this gene have been found in human patients with the autosomal recessive RP. Retinal degeneration in rd10 mice is caused by a mutation in exon 13 of the Pde6b gene. This is also a clinically relevant RP model that has a later onset and a milder retinal degeneration than that observed in the $r d 1 / \mathrm{rd} 1$ strain. $^{29}$

The $r d 1 / r d 1$ strain was used only in the time-course study experiments. All other experiments were performed using rd10 mice as a retinal degeneration model. At least four independent mice were used in each experimental condition. The numbers of mice used are indicated in the Results section and figure legends.

\section{Radiation}

The mice were anesthetized and fixated using the Magnetic Resonance Imaging-Compatible Head Fixation De- vice for mice (Narishige, Tokyo, Japan) to prevent head movement. Gamma-ray irradiation was applied using a Gammacell 40 Exactor (Nordion, Vancouver, BC, Canada) with the dose rate of the machine set at $1079 \mathrm{mGy} /$ min without a shield. Lead plates were used to weaken the radiation energy for some of the experiments. The thickness of the lead plates was 2.0 and $2.83 \mathrm{~cm}$, and the dose rates were set at 109 and $26 \mathrm{mGy} / \mathrm{min}$, respectively. Measurements were made using a Toshiba FGP-201 glass dosimeter (Toshiba, Tokyo, Japan). Sham control mice were placed in the irradiation machine cage but were not irradiated. The total dose was $650 \mathrm{mGy}$ in all of the LD groups and 2000 mGy in the high-dose (HD) groups. The LDR was $26 \mathrm{mGy} / \mathrm{min}$, the middle-dose rate was $109 \mathrm{mGy} / \mathrm{min}$, and the high-dose rate (HDR) was $1079 \mathrm{mGy} / \mathrm{min}$. The irradiation strategy is illustrated in Supplemental Figure S1, A-D (available at $h$ ttp://ajp. amjpathol.org). The initial irradiation was performed at post-natal day 10 (P10) for the rd1 mice and at P19 for rd10 mice in all experiments.

\section{Quantification of Retinal Thickness}

The mice were transcardially perfused with $4 \%$ paraformaldehyde and then the eyes were enucleated, fixed, embedded in paraffin, and processed for standard hematoxylin and eosin (H\&E) staining. A light microscope (AxioVision; Carl Zeiss Japan, Tokyo, Japan) with a 20X objective lens was used to examine each of the single sections $(4 \mu \mathrm{m})$, all of which contained a superior pole, inferior pole, optic nerve head, and entire peripheral retina. The thickness of the photoreceptor cell layer (total thickness of outer nuclear layer, photoreceptor inner segments, and photoreceptor outer segments) and the inner nuclear layer (INL) were measured in two independent fields in the mid-peripheral area within one section using the associated microscope software (AxioVision; Carl Zeiss). All values were averaged for the analysis.

\section{Terminal TUNEL Stain}

The mice were transcardially perfused with $4 \%$ paraformaldehyde and then the eyes were enucleated, fixed, and embedded in paraffin. Sections were prepared following the same method described above (quantification of retinal thickness). Appropriate sections were deparaffinized, pretreated with $80 \%$ methanol and proteinase $\mathrm{K}$, and incubated in $3 \% \mathrm{H}_{2} \mathrm{O}_{2}$. A terminal deoxynucleotidyl transferase reaction was performed for 1 hour, and labeling was detected using peroxidase-labeled antidigoxigenin and $3,3^{\prime}$-diaminobenzidine (DAB). Sections were counterstained with methyl green. The number of positive cells in the outer nuclear layer was measured in two independent fields in the mid-peripheral area within one section and then averaged for analysis. The analysis was performed by the percentage (\%) of the TUNEL-positive cells in the total cells of outer nuclear layer (ONL). 


\section{Measurement of Cone Cell Density}

Cone cell density was measured using a slight modification of a previously described method. ${ }^{29}$ Briefly, the eyecups were fixed in $4 \%$ paraformaldehyde for 30 minutes and then the entire retina was carefully dissected away from the retinal pigment epithelium. Retinas were placed in Alexa Fluor 594-conjugated lectin PNA (Molecular Probes, Tokyo, Japan) at a concentration of $5 \mu \mathrm{g} / \mathrm{mL}$ in phosphate-buffered saline (PBS) with 20\% normal goat serum for 2 hours at $4^{\circ} \mathrm{C}$. The flat-mounted retinas were examined using a confocal microscope (LSM 5 Pascal; Carl Zeiss) with a $\times 20$ objective lens. The number of cones present within four $230 \times 230-\mu m(512 \times 512$ pixels) squares located $500 \mu \mathrm{m}$ away from the center of the optic disk was counted, with four independent fields being averaged.

\section{Electroretinogram}

Cone electroretinography was performed following our previously described method. ${ }^{29}$ After overnight dark adaptation, mice were anesthetized by intraperitoneal injection of $50 \mu \mathrm{g} / \mathrm{kg}$ ketamine and $10 \mu \mathrm{g} / \mathrm{kg}$ xylazine. Electroretinograms were recorded from the corneal surface of each eye after pupil dilation (0.4\% tropicamide and $0.5 \%$ phenylephrine hydrochloride) using a gold loop corneal electrode with a light-emitting diode (Mayo Corp., Inazawa, Japan). A reference electrode was placed in the mouth, and a ground electrode was inserted into the tail. Stimuli were produced using a light-emitting diode stimulator (Mayo Corp.). The electroretinogram response signals were amplified, digitized at $10 \mathrm{kHz}$ with a band-pass filter of 0.3 to $500 \mathrm{~Hz}$, and then analyzed by a computer (PowerLab 2/25; ADInstruments, Bella Vista, Australia). After 10 minutes of light adaptation at an intensity of $25.1 \mathrm{~cd} / \mathrm{m}^{2}$, cone responses were estimated using a white stimulus of $30 \mathrm{~cd} \cdot \mathrm{s} / \mathrm{m}^{2}$. The stimulus frequency was $1 \mathrm{~Hz}$, and a total of 32 photopic measurements were averaged.

\section{PCR Array}

Total mRNA was prepared from freshly dissected whole mouse retinas using an RNeasy Kit (Qiagen, Tokyo, Japan). The mRNA was transcribed into cDNA using a $\mathrm{RT}^{2}$ First Strand Kit (SA Biosciences, Frederick, MD), and real-time PCR was performed using $\mathrm{RT}^{2}$ qPCR Master Mix (SA Biosciences). The Mouse Apoptosis $\mathrm{RT}^{2}$ Profiler PCR Array (SA Biosciences) was used for the expression analysis of the 84 key genes involved in apoptosis. All samples were normalized to five different averaged internal controls [Gusb, Hprt1, Hsp90ab1, glyceraldehyde 3-phosphate dehydrogenase (GAPDH), Actb]. Real-time PCR was performed on an ABI PRISM 7000 Sequence Detection System (Applied Biosystems, Tokyo, Japan) for 40 cycles.

\section{Standard Real-Time PCR}

Total mRNA was prepared from freshly dissected whole mouse retinas using an RNeasy Kit (Qiagen). The mRNA was transcribed using a First Strand cDNA Synthesis Kit (Applied Biosystems), and real-time PCR was performed using a TaqMan PreAmp Master Mix (Applied Biosystems). Prdx2 primers were purchased from Applied Biosystems (Assay ID: Mm00448996_m1). All samples were normalized to $\beta$-actin (Applied Biosystems). Real-time PCR was performed on an ABI PRISM 7000 Sequence Detection System (Applied Biosystems) for 40 cycles.

\section{Western Blot Analysis}

Freshly dissected whole mouse retinas were homogenized, centrifuged at $13,000 \times g$ for 10 minutes at $4^{\circ} \mathrm{C}$, and the protein concentration of the supernatant was determined using a Bradford assay. Each protein sample (40 $\mu \mathrm{g}$ ) was electrophoresed on a $5 \%$ to $20 \%$ gradient gel (ATTO, Tokyo, Japan) and transferred to a polyvinylidene fluoride membrane (ATTO). For the Prdx2 blot, a rabbit polyclonal antibody to Prdx2 (ProteinTech Group, Chicago, IL) was used followed by goat anti-rabbit IgG antibody conjugated to horseradish peroxidase (Vector Laboratories, Burlingame, CA). Protein signals were detected by chemiluminescence (Chemi-Lumi One L, Nakarai, Japan) and then exposed to imaging film. For GAPDH normalization, blots were reprobed using an antiGAPDH antibody (Sigma-Aldrich, Tokyo, Japan).

\section{In Situ Hybridization}

The probe for mouse Prdx2 was prepared by Genostaff Corp. (Tokyo, Japan), and its sequence was 5'-CACCATCAAGCCCAATGTGGATGACAGCAAGGAATACTTCTCCAAACACAACTGAGATGGGTAAACATGGGTGAGCCTGAAGCTTGGATTTCACCTGTGCCCCAACCTGGATGTCCTGTGCTGGCCCAGAAAATGCTAGATTTTCCTCCACTCTCTGAAGGGGCTGGAGTCTAGGCTGAGGTTTTCTCATTACCCACCTGGAATCTGGTGAATAGTGATCCTGCCCTGAGCACACCTAGCTGGGCCCAGGTCTATAGGAAACCA-3' . The enucleated mouse eyes were fixed with Tissue Fixative (Genostaff), embedded in paraffin, and sectioned at $4 \mu \mathrm{m}$. Sections were de-waxed, rehydrated, and fixed with $4 \%$ paraformaldehyde in PBS. After Proteinase K, refixation, and $\mathrm{HCl}$ treatment, the sections were acetylated by incubation in $0.1 \mathrm{~mol} / \mathrm{L}$ tri-ethanolamine- $\mathrm{HCl}(\mathrm{pH} 8.0)$ and $0.25 \%$ acetic anhydride for 10 minutes. Hybridization was performed with probes at $300 \mathrm{ng} / \mathrm{mL}$ in the Probe Diluent-1 (Genostaff) at $60^{\circ} \mathrm{C}$ for 16 hours. RNase treatment and blocking were performed followed by incubation with anti-DIG AP conjugate (Roche, Tokyo, Japan) for 2 hours at room temperature. Coloring reactions were performed overnight with NBT/BCIP solution (Sigma-Aldrich), and the sections were counterstained with Kernechtrot stain soIution (Mutoh, Tokyo, Japan). 


\section{Immunohistochemistry}

The mice were transcardially perfused with $4 \%$ paraformaldehyde, and the eyes were enucleated, fixed, embedded in paraffin, and sectioned at $4 \mu \mathrm{m}$. After dewaxing and rehydration, the sections were treated in a microwave for 20 minutes. Blocking was performed using Protein Block (Dako, Tokyo, Japan) for 10 minutes followed by $5 \mu \mathrm{g} / \mathrm{mL}$ rabbit polyclonal anti-Prdx2 (ProteinTech Group). Normal rabbit Ig (Dako) was used for the negative controls. The Biotin Blocking system (Dako) was used followed by anti-rabbit Ig-Biotin as the secondary antibody (Dako). Horseradish peroxidase-conjugated streptavidin (Nichirei, Tokyo, Japan) was then used followed by visualization using the DAB reaction.

\section{RNA Interference}

Intravitreal injection of siRNA targeting Prdx2 was performed following a previously described method. ${ }^{29}$ Briefly, mice were anesthetized and $0.5 \mu \mathrm{L}$ of PBS containing Prdx2 siRNA mixture was injected intravitreally using a 33-ga needle (Hamilton, Reno, NV). Three primers of siRNA targeting Prdx2 were purchased from Invitrogen Co. (Tokyo, Japan). The sequences were 5'AAAUCGGAAAGUCGGCUCCUGACUU-3', 5'-CAGAGGGAAGUACGUGGUCCUCUUU-3', and 5'-GGGUCUCUUUAUCAUCGAUGCCAAG-3'. The concentration of Prdx2 siRNA before the injection was $20 \mu \mathrm{mol} / \mathrm{L}$. A Stealth RNAi Negative Control Duplex (Invitrogen) was used as a negative control. Intravitreal injection of siRNA was performed at P18, and the mouse retina was examined at P25. LDR-LD radiation was performed 24 hours after the siRNA injection (P19).

\section{Statistical Analysis}

All results for continuous variables are expressed as the mean \pm SEM. Data between each group were compared using one-way analysis of variance followed by the Bonferroni test or unpaired $t$-test as appropriate. Values of $P<0.05$ were considered statistically significant. All analyses were performed using SPSS version 13.0 software.
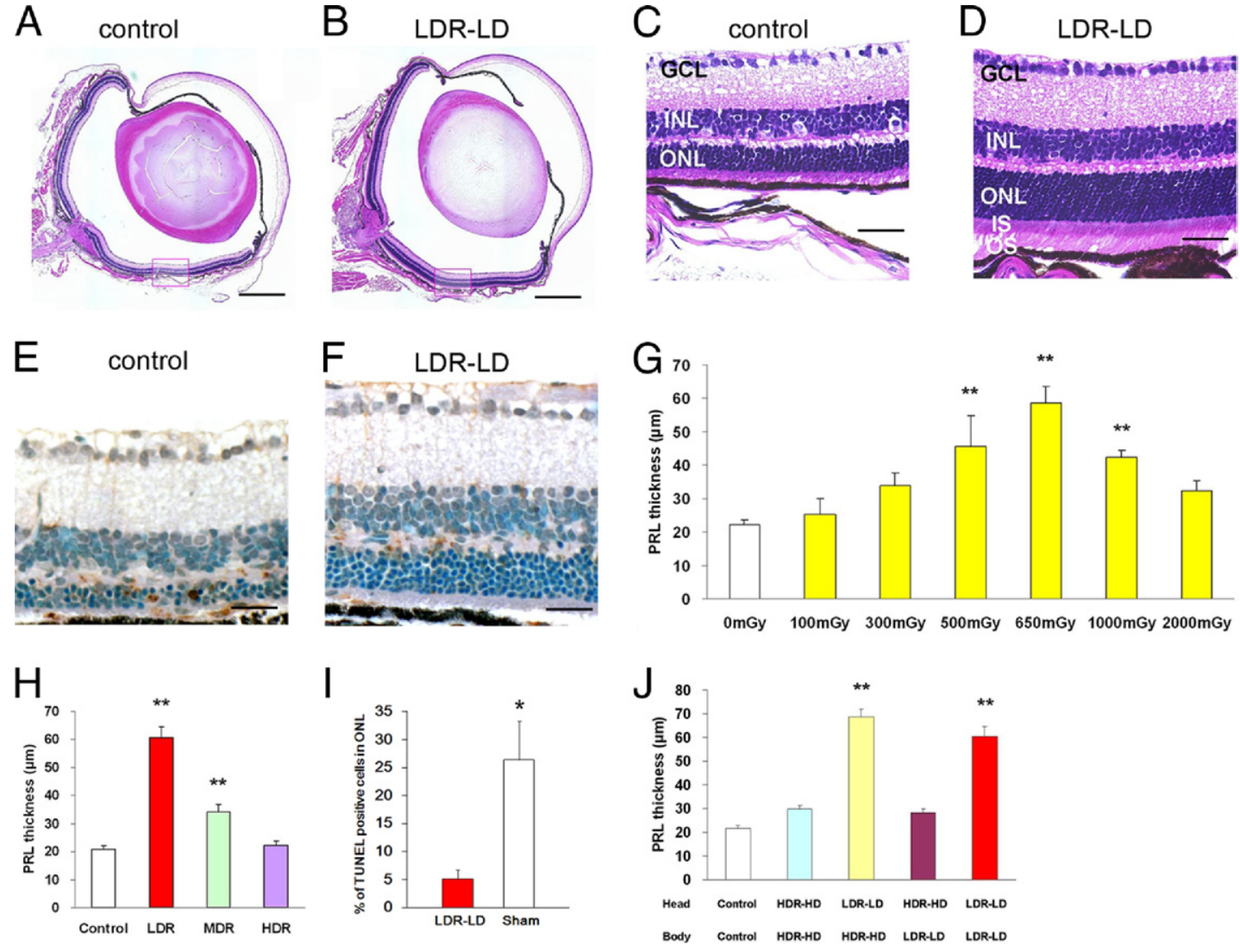

Figure 1. Antiapoptotic neuroprotection by gamma radiation depends on both the dose rate and the total dose. A and B: Whole eye (P25) sections from nonirradiated (A) and from low-dose-rate, low-dose (LDR-LD) irradiated (P19) (B) rd10 mice. Scale bar $=480 \mu \mathrm{m}$. C and D: Magnified images of the red box in figures (A) and (B). GCL, ganglion cell layer; INL, inner nuclear layer; IS, inner segment of the photoreceptor cell; ONL, outer nuclear layer; OS, outer segment of the photoreceptor cell. Scale bar $=40 \mu \mathrm{m}$. E and F: Terminal deoxynucleotidyl transferase-mediated dUTP nick end-labeling (TUNEL) staining section of the nonirradiated $(\mathbf{E})$ and of the LDR-LD-irradiated $(\mathbf{F})$ retina of $r d 10$ (P24) mice. Scale bar $=30 \mu \mathrm{m}$. G: Dose-response relationship in LDR radiation (dose rate: 26 $\mathrm{mGy} /$ minute). PRL, photoreceptor layer (ONL + IS + OS) ( $n=4$ to 6 per group). H: Dose-rate-response relationship. Total dose was 650 mGy in all groups. HDR, high-dose rate (1079 $\mathrm{mGy} / \mathrm{min}$ ) ( $n=8$ to 12 per group); LDR, low-dose rate (26 mGy/min); MDR, middle-dose rate (109 mGy/min). I: Percentage (\%) of the TUNEL-positive cells in the ONL ( $n=6$ per group). J: The neuroprotective effect when the head or the body of the mice is protected by lead plates ( $n=$ 6 to 10 per group). HD, high-dose (2000 mGy total); LD, low-dose (650 mGy total). The radiation dose in the protected part is LDR-LD, whereas that of the other part is HDR-HD. A detailed strategy is presented in Supplemental Figure S1 (available at http://ajp.amjpathol.org). ${ }^{*} P<0.05,{ }^{* * *} P<0.01$ versus control. $P$ values were from post hoc analysis. 


\section{Results}

\section{Antiapoptotic Neuroprotection by Gamma Radiation Depends on Both Dose Rate and Total Dose}

To study the effects of LD radiation on neurodegeneration, various doses of total-body LD radiation were administered in the retinal degeneration mouse model, rd10 (B6.CXB1-Pde6b $b^{r d 10} / \mathrm{J}$; Jackson Laboratory) (Figure 1). This mouse strain has a mutation in exon 13 of the $\beta$-subunit of the rod cGMP phosphodiesterase, a mutation that is relevant to a type of human retinitis pigmentosa. In the rd10 mouse strain, the photoreceptor cells begin to die by apoptosis; on P25, the outer nuclear layer was shortened to only two to three rows (Figure 1, A and C). However, if these mice were irradiated on P19 with a dose $<2000 \mathrm{mGy}$, this treatment dramatically prevented photoreceptor cell death (Figure 1, B and D). A 650 mGy dose had the most powerful rescue effect, and when the total dose was increased, the effect gradually decreased (Figure 1G). This dose-response curve is consistent with the hypothetical curve that depicts hormesis (" $\cap$ "-shape). ${ }^{30}$

In addition to the dose-response effect, we also tested the effect of the radiation dose rate. Photoreceptor sur- vival was much higher after the LDR (26 mGy/min) than the HDR (1079 $\mathrm{mGy} / \mathrm{min}$ ) and middle-dose rate (109 $\mathrm{mGy} / \mathrm{min}$ ) (Figure $1 \mathrm{H}$ ). These results suggest that, not only the total dose, but also the dose rate, can greatly affect the neuroprotective effects induced by ionizing radiation. Therefore, the remaining LDR-LD experiments were performed using a dose rate of $26 \mathrm{mGy} / \mathrm{min}$ and a total dose of $650 \mathrm{mGy}$. LDR-LD irradiation decreased the percentage of the TUNEL-positive cells in the ONL of P21 rd10 mice (Figure 1, E, F, and I). LDR-LD radiation prevents the photoreceptor apoptosis that is normally seen in inherited retinal degeneration.

We previously reported that the mobilization of hematopoietic progenitors can rescue retinal degeneration. ${ }^{29,31}$ Because LD radiation can induce hematopoietic progenitor cell mobilization into the peripheral blood circulation, $^{32}$ it is possible that the neuroprotective effect induced by ionizing radiation exposure originates from indirect effects on the body rather than a direct retinal effect. In our experiments, neuroprotection was observed when the head was exposed to LDR-LD radiation regardless of the radiation dose administered to the body (Figure 1J). Thus, the antiapoptotic effect of LDR-LD might be due to the direct effect on the retina. The irradiation scheme is illustrated in Supplemental Figure S1 (available at http://ajp.amjpathol.org).
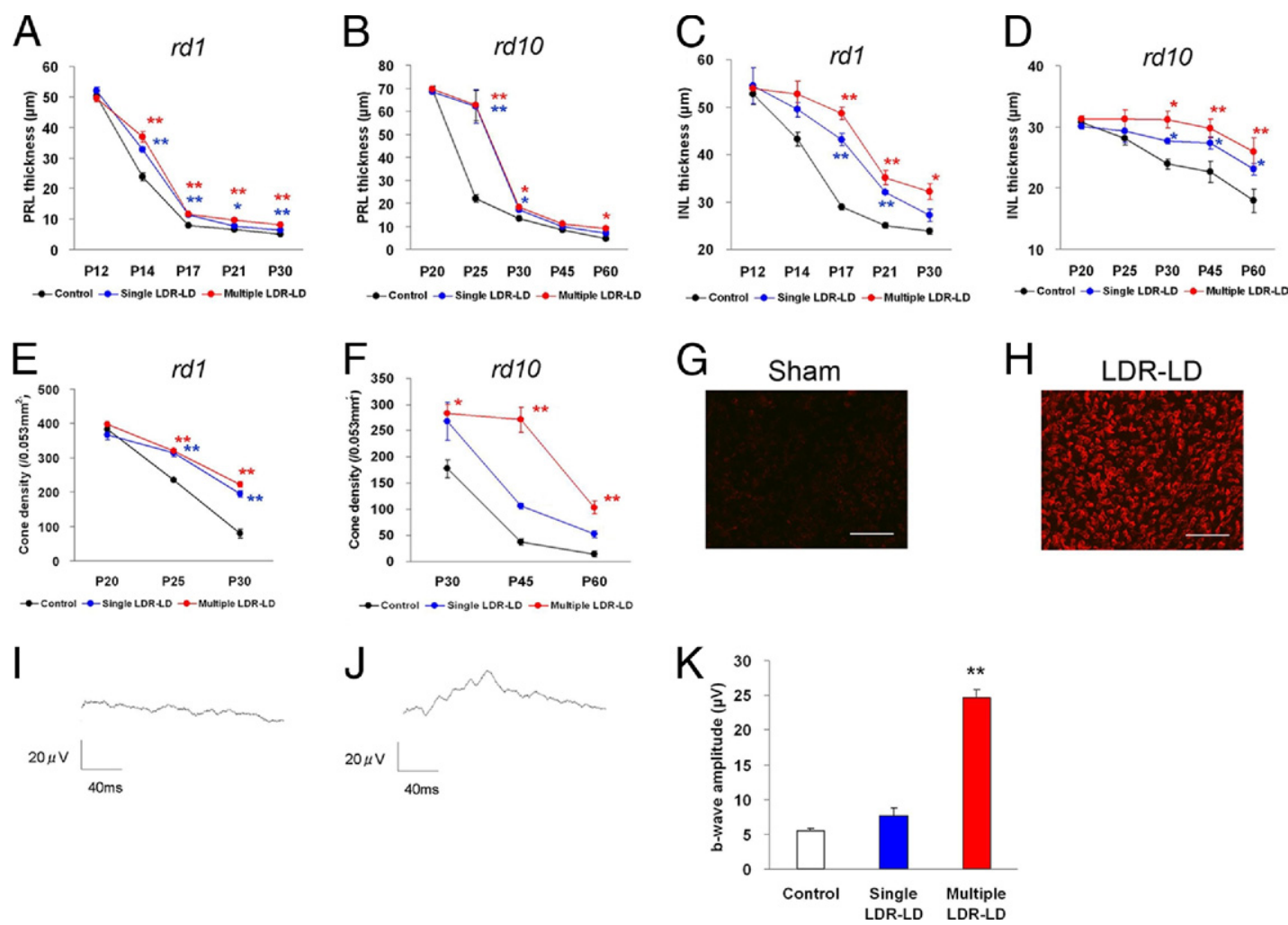

Figure 2. Analysis of the neuroprotective effects of low-dose-rate, low-dose (LDR-LD) radiation for duration, genetic differences, and irradiation times. Multiple irradiations were performed on P10 to P14 once per day in the $r d 1$ mouse strain and on P19, P25, P30, P35, P40, P45, P50, and P55 in the $r d 10$ mouse strain. A-D: Time-course of photoreceptor layer thickness ( $\mathbf{A}$ and $\mathbf{B})$ and INL thickness $(\mathbf{C}$ and $\mathbf{D})$ in $r d 1$ mice $(\mathbf{A}$ and $\mathbf{C})(n=6$ to 12 per point) and $r d 10$ mice $(\mathbf{B}$ and $\mathbf{D})(n=4$ to 8 per point). $\mathbf{E}$ and $\mathbf{F}$ : Time-course of cone cell density in $r d 1$ (E) $(n=6$ per point $)$ and $r d 10$ (F) $(n=8$ to 16 per point) mice. $\mathbf{G}$ and $\mathbf{H}$ : Flat-mount image of retinal cone photoreceptor cells stained with lectin PNA (red). Nonirradiated retina (G) and LDR-LD-irradiated retina (H) from $r d 10$ (P45) mice. Scale bars: $50 \mu \mathrm{m}$. I and $\mathbf{J}$ : Cone electroretinogram. Nonirradiated retina (I) and LDR-LD-irradiated retina (J) of $r d 10$ (P45) mice. K: B-wave amplitude of the cone electroretinogram in $r d 10(\mathrm{P} 45)$ mice $(n=8) .{ }^{*} P<0.05,{ }^{* *} P<0.01$ versus control. $P$ values were from post hoc analysis. 
Analyses of Neuroprotective Effects by LDR-LD Radiation for the Duration, Genetic Difference, and Irradiation Times

We subsequently examined whether the neuroprotection induced by LDR-LD radiation was present in other inherited retinal degeneration mouse models. This question is of importance since human inherited retinal degeneration originates from many kinds of gene mutations and mutation patterns ( $>40$ genes). ${ }^{33}$ Furthermore, since human inherited retinal degeneration often shows gradual progression over time, we also examined the hypothesis that the protective effects could be enhanced by multiple irradiations. A single irradiation was performed on P10 in the rd1 mouse strain ${ }^{31}(\mathrm{C} 3 \mathrm{H} / \mathrm{He}$; the Jackson Laboratory) and on P19 in the rd10 mouse strain. Multiple irradiations were performed on P10 to P14 once per day in the rd1 strain and on P19, P25, P30, P35, P40, P45, P50, and P55 in the $r d 10$ strain. Although the LDR-LD radiation promoted photoreceptor survival in both the rd1 and rd10 mice, the duration of this protective effect was limited, even when multiple irradiations were performed (Figure 2, $A$ and $B$ ). LDR-LD radiation also showed a protective effect against secondary degeneration in the INL where the nuclei of the bipolar, horizontal, amacrine, and Muller cells exist; interestingly, this effect lasted much longer than it did for primary photoreceptor degeneration in both the $r d 1$ and $r d 10$ mouse strains. Moreover, this rescue effect was enhanced by multiple irradiations (Figure 2, C and D). The cone cells are clinically important in that they are the key to maintaining patients' visual acuity. Therefore, we also examined the effect of the LDR-LD radiation on the cone photoreceptor cell degeneration. Cone cell density as measured by lectin-PNA staining in mounted retinal tissue was markedly preserved by LDR-LD radiation in both the rd1 and rd10 mouse strains (Figure 2, $\mathrm{E}-\mathrm{H}$ ). This preservation proved to be a long-term protective effect, especially when induced by multiple irradiations. The functional response of the cone cells as measured by an electroretinogram was also preserved by LDR-LD radiation (Figure 2, I-K). Although these results indicate that there is a limited time span of the LDR-LD radiation rescue effect for the rod cell degeneration that is directly associated with genetic mutation, there is a dramatic effect on secondary degeneration of the INL and cone photoreceptors. Thus, enhancing the rescue effect through the use of multiple irradiations may be a therapeutic strategy that can be used for human inherited retinal degeneration regardless of the underlying genetic defects.

\section{Prdx2 Plays a Major Role in the Neuroprotective Effects Induced by LDR-LR Radiation}

The PCR array for the apoptosis pathway was designed to simultaneously examine mRNA expression levels for the 84 genes involved in apoptosis. ${ }^{34}$ Compared to the sham-

HDR-HD radiation

\begin{tabular}{|c|c|c|c|c|c|c|c|c|c|c|c|}
\hline Akt1 & Apaf1 & Api5 & Att5 & Bad & Bag1 & Bag3 & Bak1 & Bax & Bcl10 & Bcl2 & Bcl211 \\
\hline Bc12110 & Bcl212 & Bid & Birc1a & Birc1b & Birc2 & Birc3 & Birc4 & Birc5 & Bnip2 & Bnip3 & Bnip31 \\
\hline Bok & Card10 & Nod1 & Card6 & Casp1 & Casp12 & Casp14 & Casp2 & Casp3 & Casp4 & Casp6 & Casp7 \\
\hline Casp8 & Casp9 & Cflar & Cidea & Cideb & Cradd & Dad1 & Dapk1 & Dffa & Dffb & Tsc22d3 & Fadd \\
\hline Fas & Fas1 & Hells & II10 & Lhx4 & Ltbr & Mcl1 & Nfkb1 & Nme5 & Nol3 & Pak7 & Pim2 \\
\hline Polb & Prdx2 & Pycard & Ripk1 & Rnf7 & Sphk2 & Tnf & Tnfrs110b & Tnfrsf11b & Tnfrsf1a & Cd40 & Tnfsf10 \\
\hline Tnfsf12 & Cd40lg & Cd70 & Traf1 & Traf2 & Traf3 & Trp53 & Trp53bp2 & Trp53inp1 & Trp63 & Trp73 & Zc3hc1 \\
\hline
\end{tabular}

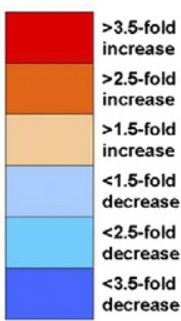

B

LDR-LD radiation

\begin{tabular}{|c|c|c|c|c|c|c|c|c|c|c|c|}
\hline Akt1 & Apaf1 & Api5 & Atf5 & Bad & Bag1 & Bag3 & Bak1 & Bax & Bcl10 & Bcl2 & Bcl211 \\
\hline Bc12110 & Bc1212 & Bid & Birc1a & Birc1b & Birc2 & Birc3 & Birc4 & Birc5 & Bnip2 & Bnip3 & Bnip31 \\
\hline Bok & Card10 & Nod1 & Card6 & Casp1 & Casp12 & Casp14 & Casp2 & Casp3 & Casp4 & Casp6 & Casp7 \\
\hline Casp8 & Casp9 & Cflar & Cidea & Cideb & Cradd & Dad1 & Dapk1 & Dffa & Dffb & Tsc22d3 & Fadd \\
\hline Fas & Fas1 & Hells & I110 & Lhx4 & Ltbr & Mcl1 & Nfkb1 & Nme5 & Nol3 & Pak7 & Pim2 \\
\hline Polb & Prdx2 & Pycard & Ripk1 & Rnf7 & Sphk2 & Tnf & Tnfrsf10b & Tnfrsf11b & Tnfrsf1a & Cd40 & Tnfsf10 \\
\hline Tnff112 & Cd40lg & Cd70 & Traf1 & Traf2 & Traf3 & Trp53 & Trp53bp2 & Trp53inp1 & Trp63 & Trp73 & Zc3hc1 \\
\hline
\end{tabular}

Figure 3. Comparison of apoptosis-associated gene expression pattern between high-dose-rate- and low-dose-rate-irradiated retinas. A and B: Apoptosis-related mRNA expression collected from irradiated $r d 10$ retinas ( 2 hours after radiation) $(n=4)$. HDR-HD, high-dose-rate high-dose (1079 mGy/min; total $2000 \mathrm{mGy})$; LDR-LD, low-dose-rate, low-dose $(26 \mathrm{mGy} / \mathrm{min}$; total $650 \mathrm{mGy}$ ). Antiapoptotic genes (italic font) are surrounded by red squares, and apoptosis-inducible genes are in black squares. 
treated control retina (P19), Prdx2 was highly up-regulated (563\%) in the LDR-LD-irradiated (26 mGy/min; total 650 mGy) retina, but not in the HDR-HD irradiated (1079 mGy/ min; total 2000 mGy) retina (Figure 3, A and B). In addition, the apoptosis inducible genes caspase- 3 and caspase- 7 were also up-regulated in the HDR-HD-irradiated retina, suggesting that $\mathrm{HD}$ treatment was too much for the retinal cells to handle, and thus, they were not able to survive. Prdx2 is a member of the peroxiredoxin family and an antioxidative gene. ${ }^{26}$ Oxidative stress reportedly plays an important role in the pathogenesis of inherited retinal degeneration. ${ }^{35}$ Moreover, recent studies strongly suggest that Prdx2 plays a neuroprotective role in other neurodegenerative diseases. ${ }^{27,28}$ Therefore, we hypothesized that Prdx2 up-regulation due to LDR-LD radiation increases the antioxidative ability of the retinal cells and may counteract the photoreceptor degeneration stress, thereby re- sulting in the neuroprotective effect. The induction of Prdx2 began 1 hour after irradiation, peaked at 2 hours (463\%), and returned to normal levels by 24 hours (Figure 4A). Western blot analysis revealed that the Prdx2 protein increased 1 hour after irradiation, with the increased levels continuing even after the 24-hour examination period (Figure 4B). In the normal retina, Prdx2 mRNA was expressed in the inner retina, especially in the ganglion cells and in the cells of the inner nuclear layer (Figure 4, C-F). Surprisingly, in the LDR-LD-irradiated retina, there was new expression of Prdx2 mRNA in the photoreceptor cells of the outer nuclear layer, especially within the inner segment of the photoreceptors (Figure 4, C-F).

Immunohistochemical analysis further confirmed the results of an in situ hybridization study (Figure 4, G-J). These results strongly indicate that LDR-LD radiation induces Prdx2 up-regulation in photoreceptor cells.
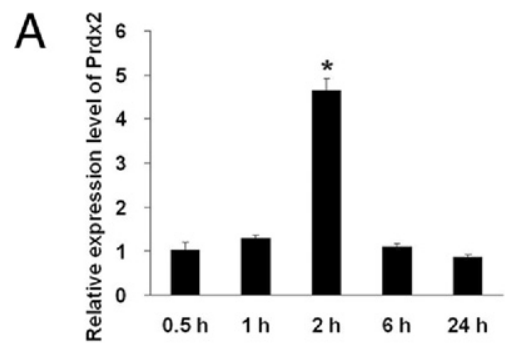

C

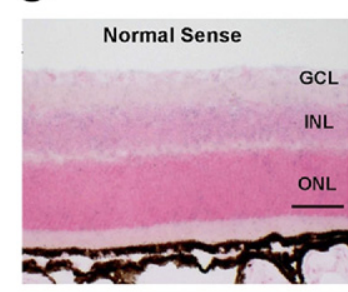

D

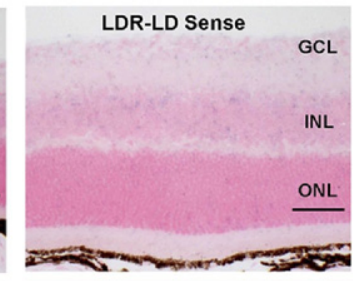

$\mathrm{G}$

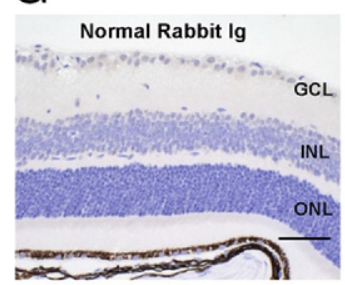

$\mathrm{H}$

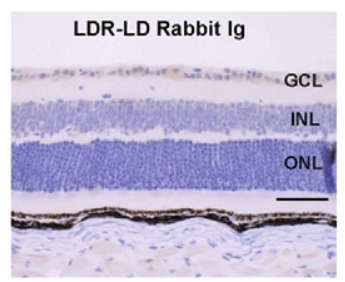

$\mathrm{K}$

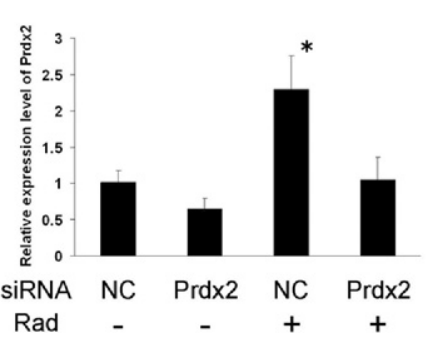

B

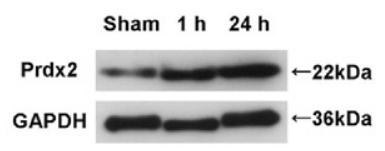

E

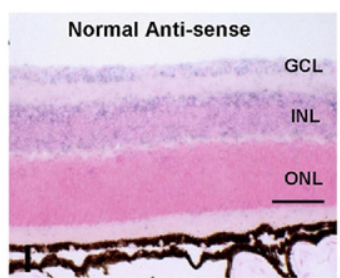

$\mathrm{F}$
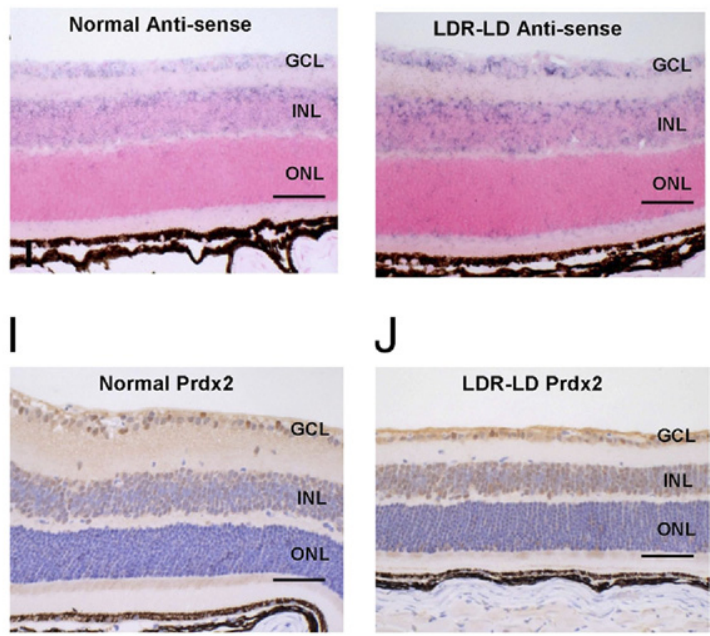

$\mathrm{J}$

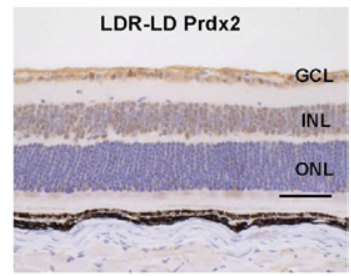

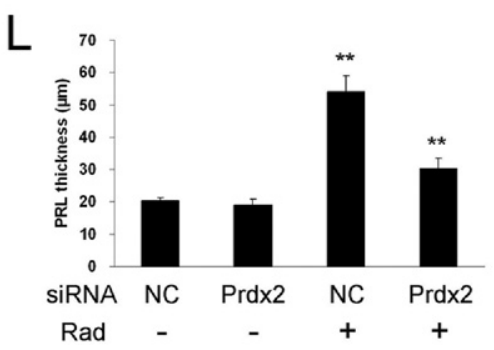

Figure 4. $\operatorname{Prd} x 2$ plays a major role in the neuroprotective effects induced by low-dose-rate, low-dose (LDR-LD) radiation. A: Time course of $\operatorname{Prd} x 2$ mRNA levels in LDR-LD-irradiated retinas $(n=6)$. B: Western blot analysis of $\operatorname{Prd} 22$ in LDR-LD-irradiated retinas $(n=9)$. C-F: $I n$ situ hybridization study of $P r d x 2$ in the retina. G-J: Immunohistochemistry of $\operatorname{Prd} x 2$ in the retina. K: Relative expression level of Prdx2 mRNA 26 hours after intravitreal siRNA injection. Radiation was administered at 24 hours after injection. NC, negative control injected; Rad, LDR-LD radiation $(n=12)$. A normal C57BL/6 strain was used in experiments A to K. L: PRL thickness of an $r d 10$ (P25) mouse retina. Intravitreal injection of siRNA at P18. LDR-LD radiation was performed 24 hours after the siRNA injection (P19) $(n=12)$. Scale bars: $60 \mu \mathrm{m}(\mathbf{C}-\mathbf{J}) .{ }^{*} P<0.05,{ }^{* *} P<0.01$ versus control. $P$ values were from post hoc analysis. 
To determine whether LDR-LD irradiation-induced protection of photoreceptors is truly mediated by $\operatorname{Prd} \times 2 \mathrm{up}$ regulation, $\operatorname{Prd} \times 2$ was silenced by an intravitreal injection of siRNA that targeted it. The Prdx2 siRNA sufficiently silenced the expression and up-regulation of Prdx2 mRNA levels in the injected retinas (Figure 4K). In contrast to the negative control-injected eyes that exhibited marked photoreceptor (P25) preservation, Prdx2 siRNAinjected eyes showed a much lesser photoreceptor rescue effect (Figure 4L). These data strongly indicate that $\operatorname{Prd} \times 2$ up-regulation within the photoreceptor cells is the key mechanism for the LDR-LD-induced neuroprotective action. The expression of Prdx2 in the rd10 retina and the effect of siRNA silencing were confirmed by immunohistochemistry (see Supplemental Figure S1, E-G, at $h t t p: / /$ ajp.amjpathol.org).

\section{Discussion}

These data are the first to demonstrate that LD ionizing radiation has a protective effect for retinal photoreceptor cells. In one study that reported a beneficial effect on retinal ganglion cells, ${ }^{36}$ a much higher dose (10 Gy) was applied and the underlying mechanisms were not investigated. Although the effect of the LD and LDR settings (650 mGy, $26 \mathrm{mGy} / \mathrm{min}$ ) used in this study on retinal ganglion cells has not yet been explored, it appears that the underlying mechanisms providing neuroprotection differ between the two studies. The degeneration speed in our retinal degeneration models is much faster than that in the glaucoma model used in the earlier study. ${ }^{36}$ This is a great advantage and allows for the calculation of a dose-response curve and the peak dose of the protective effect (Figure 1G). The dose-response curve of our data showed the characteristic inverted U-shape that is typically observed in other hormetic phenomena. ${ }^{30}$ We therefore suggest that our findings are a kind of "adaptive response" or "neurohormesis" 2 of the retinal photoreceptor cells to very low-dose radiation.

Immunohistochemical and silencing RNA analyses strongly indicated that induction of antioxidative protein Prdx2 in photoreceptor cells by ionizing radiation is one of the molecular mechanisms underlying neurohormesis. $\operatorname{Prd} \times 2$ is a member of the peroxiredoxin family that plays an antioxidant protective role by modulating $\mathrm{H}_{2} \mathrm{O}_{2}$. $\operatorname{Prd} 22$ shows multiple cellular functions, including protection of proteins and lipids against oxidative injury, controlling cellular proliferation or differentiation, and mediates the intracellular apoptosis signaling pathways. ${ }^{37}$ Although Prdx2 expression has been observed in many tissues and cells, little is known of Prdx2 regulation details other than oxidative stress.

A recent study reported that forkhead box $\mathrm{O} 3 \mathrm{a}$ (Foxo3a) of the forkhead family of transcription factors regulates Prdx2 expression in the trabecular meshwork cells of the eye. ${ }^{38}$ Another study reported that nuclear Prdx2 prevents cancer cell death induced by DNA-damaging agents independent of its antioxidative activity and suggested that Prdx2 might be the first antioxidant enzyme involved in the DNA repair process. ${ }^{39}$ We did not study the antioxidative expression of other genes such as superoxide dismutases, which are reportedly involved in radiation hormesis seen in other nonneural tissues. ${ }^{24}$

Transcription factors that regulate neurohormesis, such as nuclear factor E2-related factor (NRF2) or cyclic adenosine monophosphate-response element-binding protein has been reported. ${ }^{39,40}$ Transcriptions of some of the peroxiredoxin family members is regulated by the NRF2-Keap1 system, ${ }^{41}$ but the relationship between Prdx2 and NRF2 remains under investigation.

These data pave the way for possible neuroprotective therapies that can be used for treating vision-threatening retinal diseases, including inherited retinal degeneration. Focal irradiation has long been performed as an ocular disease therapy to avoid the adverse effects of HD radiation. Age-related macular degeneration is one of the main targets of focal irradiation therapy. ${ }^{42}$ The concept of therapy for age-related macular degeneration is to damage the cells comprising neovascularization using $\mathrm{HD}$ radiation with the aim of occluding the vasculature and inactivating the pathological formation of new vessels. The new therapeutic concept using focal irradiation suggested by our data are entirely different. The hormetic effects induced by very low-dose radiation in the residual retinal cells counter the disease stress and make progression very slow. This is a nondestructive therapy, and the fact that multiple irradiation rounds further strengthen the neuroprotective effect including functional rescue (Figure 2) gives us hope for future clinical applications. The different genetic mutations that occur for rhodopsin, arrestin, phosphodiesterase, and others result in many types of the inherited form of human retinal degeneration. If these degeneration types can be rescued by LDR-LD irradiation through an antioxidative mechanism, diseased human retinas will most likely be protected regardless of the degeneration mechanism involved. In addition to retinal degeneration, oxidative stress has been implicated in the progression of other neurodegenerative diseases. ${ }^{43}$ We anticipate that LDR-LD radiation will be a novel therapeutic concept for various kinds of neurodegeneration with the aim of increasing antioxidative ability in the degenerating cells and preventing oxidative stress-related cell death.

\section{Acknowledgments}

We thank Dr. Manabu Sasahara and Atsuko Urasaki for their contributions to this work.

\section{References}

1. Calabrese EJ, Baldwin LA: Defining hormesis. Hum Exp Toxicol 2002, 21:91-97

2. Mattson MP, Cheng A: Neurohormetic phytochemicals: low-dose tox ins that induce adaptive neuronal stress responses. Trends Neurosci 2006, 29:632-639

3. Kirino T: Ischemic tolerance. J Cereb Blood Flow Metab 2002, 22 : 1283-1296

4. Jiang X, Zhu D, Okagaki P, Lipsky R, Wu X, Banaudha K, Mearow K, Strauss KI, Marini AM: N-methyl-d-aspartate, TrkB receptor activation in cerebellar granule cells: an in vitro model of preconditioning to 
stimulate intrinsic survival pathways in neurons. Ann N Y Acad Sci 2003, 993:134-145; discussion 159-160

5. Ding Y, Li J, Luan X, Ding YH, Lai Q, Rafols JA, Phillis JW, Clark JC, Diaz FG: Exercise pre-conditioning reduces brain damage in ischemic rats that may be associated with regional angiogenesis and cellular overexpression of neurotrophin. Neuroscience 2004, 124: 583-591

6. Mattson MP: Energy intake, meal frequency, and health: a neurobiological perspective. Annu Rev Nutr 2005, 25:237-260

7. Huang PL: Nitric oxide and cerebral ischemic preconditioning. Cell Calcium 2004, 36:323-329

8. Dore S: Decreased activity of the antioxidant heme oxygenase enzyme: implications in ischemia and in Alzheimer's disease. Free Radic Biol Med 2002, 32:1276-1282

9. Jiang X, Tian F, Mearow K, Okagaki P, Lipsky RH, Marini AM: The excitoprotective effect of $\mathrm{N}$-methyl-d-aspartate receptors is mediated by a brain-derived neurotrophic factor autocrine loop in cultured hippocampal neurons. J Neurochem 2005, 94:713-722

10. See V, Boutillier AL, Bito H, Loeffler JP: Calcium/calmodulin-dependent protein kinase type IV (CaMKIV) inhibits apoptosis induced by potassium deprivation in cerebellar granule neurons. FASEB J 2001 , 15:134-144

11. Luckey TD: Physiological benefits from low levels of ionizing radiation. Health Phys 1982, 43:771-789

12. Yonezawa M, Misonoh J, Hosokawa $Y$ : Two types of X-ray-induced radioresistance in mice: presence of 4 dose ranges with distinct biological effects. Mutat Res 1996, 358:237-243

13. Lorenz E, Hollcroft JW, Miller E, Congdon CC, Schweisthal R: Longterm effects of acute and chronic irradiation in mice. I. Survival and tumor incidence following chronic irradiation of $0.11 \mathrm{r}$ per day. J Natl Cancer Inst 1955, 15:1049-1058

14. Ducoff HS: Form of the increased longevity of Tribolium after X-irradiation. Exp Gerontol 1975, 10:189-193

15. Mine M, Okumura Y, Ichimaru M, Nakamura T, Kondo S: Apparently beneficial effect of low to intermediate doses of A-bomb radiation on human lifespan. Int J Radiat Biol 1990, 58:1035-1043

16. Anderson RE, Lefkovits I: In vitro evaluation of radiation-induced augmentation of the immune response. Am J Pathol 1979, 97:456472

17. Kojima S, Ishida H, Takahashi M, Yamaoka K: Elevation of glutathione induced by low-dose gamma rays and its involvement in increased natural killer activity. Radiat Res 2002, 157:275-280

18. Ina $Y$, Sakai K: Prolongation of life span associated with immunological modification by chronic low-dose-rate irradiation in MRL-lpr/lp mice. Radiat Res 2004, 161:168-173

19. Miyachi $Y$, Kasai $H$, Ohyama $H$, Yamada $T$ : Changes of aggressive behavior and brain serotonin turnover after very low-dose X-irradiation of mice. Neurosci Lett 1994, 175:92-94

20. Dobbs HJ, Barrett A, Rostom AY, Peckham MJ: Total-body irradiation in advanced non-Hodgkin's lymphoma. Br J Radiol 1981, 54:878881

21. Jacobs P, King HS: A randomized prospective comparison of chemotherapy to total body irradiation as initial treatment for the indolent lymphoproliferative diseases. Blood 1987, 69:1642-1646

22. Shen RN, Lu L, Kaiser HE, Broxmeyer HE: Murine AIDS cured by low dosage total body irradiation. Adv Exp Med Biol 1997, 407:451-458

23. Hashimoto S, Shirato H, Hosokawa M, Nishioka T, Kuramitsu $Y$, Matushita K, Kobayashi M, Miyasaka K: The suppression of metastases and the change in host immune response after low-dose totalbody irradiation in tumor-bearing rats. Radiat Res 1999, 151:717-724

24. Takahashi M, Kojima S, Yamaoka K, Niki E: Prevention of type diabetes by low-dose gamma irradiation in NOD mice. Radiat Res 2000, 154:680-685
25. Luckey TD: Radiation Hormesis. Boca Raton, FL, CRC Press, 1991

26. Wood ZA, Schroder E, Robin Harris J, Poole LB: Structure, mechanism and regulation of peroxiredoxins. Trends Biochem Sci 2003 , 28:32-40

27. Fang J, Nakamura T, Cho DH, Gu Z, Lipton SA: S-nitrosylation of peroxiredoxin 2 promotes oxidative stress-induced neuronal cell death in Parkinson's disease. Proc Natl Acad Sci U S A 2007, 104: 18742-18747

28. Krapfenbauer K, Engidawork E, Cairns N, Fountoulakis M, Lubec G: Aberrant expression of peroxiredoxin subtypes in neurodegenerative disorders. Brain Res 2003, 967:152-160

29. Sasahara M, Otani A, Oishi A, Kojima H, Yodoi Y, Kameda T, Nakamura $\mathrm{H}$, Yoshimura N: Activation of bone marrow-derived microglia promotes photoreceptor survival in inherited retinal degeneration. Am J Pathol 2008, 172:1693-1703

30. Calabrese EJ, Baldwin LA: Toxicology rethinks its central belief. Nature 2003, 421:691-692

31. Otani A, Dorrell MI, Kinder K, Moreno SK, Nusinowitz S, Banin E, Heckenlively J, Friedlander $M$ : rescue of retinal degeneration by intravitreally injected adult bone marrow-derived lineage-negative hematopoietic stem cells. J Clin Invest 2004, 114:765-774

32. Li W, Wang G, Cui J, Xue L, Cai L: Low-dose radiation (LDR) induces hematopoietic hormesis: IDR-induced mobilization of hematopoietic progenitor cells into peripheral blood circulation. Exp Hematol 2004 32:1088-1096

33. Hartong DT, Berson EL, Dryja TP: Retinitis pigmentosa. Lancet 2006, 368:1795-1809

34. Zhan Q, Signoretti S, Whitaker-Menezes D, Friedman TM, Korngold R, Murphy G-F: Cytokeratin15-positive basal epithelial cells targeted in graft-versus-host disease express a constitutive antiapoptotic phenotype. J Invest Dermatol 2007, 127:106-115

35. Komeima K, Rogers BS, Lu L, Campochiaro PA: Antioxidants reduce cone cell death in a model of retinitis pigmentosa. Proc Natl Acad Sci U S A 2006, 103:11300-11305

36. Anderson MG, Libby RT, Gould DB, Smith RS, John SW: High-dose radiation with bone marrow transfer prevents neurodegeneration in an inherited glaucoma. Proc Natl Acad Sci U S A 2005, 102:45664571

37. Jeong WS, Jun M, Kong AN: Nrf2: a potential molecular target for cancer chemoprevention by natural compounds. Antioxid Redox Signal 2006, 8:99-106

38. Kang SW, Chae HZ, Seo MS, Kim K, Baines IC, Rhee SG: Mammalian peroxiredoxin isoforms can reduce hydrogen peroxide generated in response to growth factors and tumor necrosis factor- $\alpha$. J Biol Chem 1998, 273:6297-6302

39. Miyamoto N, Izumi $H$, Miyamoto R, Kubota T, Tawara A, Sasaguri $Y$, Kohno K: Nipradilol and timolol induce Foxo3a and peroxiredoxin 2 expression and protect trabecular meshwork cells from oxidative stress. Invest Ophthalmol Vis Sci 2009, 50:2777-278440

40. West AE, Chen WG, Dalva MB, Dolmetsch RE, Kornhauser JM, Shaywitz AJ, Takasu MA, Tao X, Greenberg ME: Calcium regulation of neuronal gene expression. Proc Natl Acad Sci U S A 2001, 98:1102411031

41. Ishii T, Yanagawa T: Stress-induced peroxiredoxins. Subcell Biochem 2007, 44:375-384

42. Mandai M, Takahashi M, Miyamoto $H$, Hiroshiba N, Kimura H, Ogura $Y$, Honda $Y$, Sasai K: Long-term outcome after radiation therapy for subfoveal choroidal neovascularization associated with age-related macular degeneration. Jpn J Ophthalmol 2000, 44:530-537

43. Barnham KJ, Masters CL, Bush Al: Neurodegenerative diseases and oxidative stress. Nat Rev Drug Discov 2004, 3:205-214 